\title{
LEADERSHIP STYLE, INTERPERSONAL RELATIONSHIPS AND THE QUALITY OF THE MEDICAL ACT DURING THE COVID-19 PANDEMIC WITHIN A STATE HOSPITAL
}

\author{
Răzvan-Andrei CORBOȘ ${ }^{a}$, Daniel Alin CRISTIAN ${ }^{b}$, Ruxandra-Irina POPESCU, \\ Ovidiu-Iulian BUNEA $A^{d}$, Răzvan SC $\breve{A} U N A S U^{e}$ \\ ${ }^{\mathrm{a}, \mathrm{c}, \mathrm{d}}$ Bucharest University of Economic Studies, Romania \\ b,e UMF Carol Davila, Bucharest, Romania
}

\begin{abstract}
Through this research approach, we aimed to identify the main characteristics of the leaders of the Coltea Clinical Hospital, in the conditions and context of the COVID-19 pandemic. We chose this approach in view of the fact that the pandemic was and still is a challenge for all medical staff in the medical unit, which could have led to changes in behaviors, interpersonal relationships, with potential influences on the medical act. The results indicate that within the medical unit there is a rather transformational leadership style, with very little transactional influences, perceptions of interpersonal relationships are positive, and in terms of the quality of the medical act the highest scores were obtained by equity of the medical services and the item that described patient safety, all being elements that can characterize the activity of medical units during the COVID-19 pandemic.
\end{abstract}

KEYWORDS: Leadership, Health Organization, Quality, COVID-19.

DOI: 10.24818/IMC/2021/04.14

\section{INTRODUCTION}

Studies indicate multiple aspects according to which the quality of medical services lies in the quality of management and leadership of professionals providing health care services (Sfantou et al., 2017). Leadership has many definitions in the literature that describe the concept as referring to the relationship between people who lead and people who choose to be led, through behaviors that involve organizing and coordinating the activities of a group of individuals in order to achieve a common goal (Al-Sawai, 2013). Leadership manifests itself in various forms called leadership styles, and six of them are widely noted: transformational, transactional, laissez-faire, autocratic, goal-oriented, and relationship-oriented. However, our work is oriented towards the analysis of leadership style more in terms of the transformational and transactional style to the detriment of others, as similar studies in the literature have revealed their stronger influences in medical organizations.

Transformational leadership is characterized by an emphasis on relationships and motivation within team or group members. Leaders who show transformational traits often succeed in inspiring trust, gaining the respect of others, but also their loyalty through a common vision, all resulting in higher levels of productivity and morale. On the other hand, the transactional leader behaves as a change manager through various exchanges he/she makes with employees that are meant to improve productivity and efficiency (Sfantou et al., 2017). Transformational leadership is considered to be the most effective leadership style when it comes to organizations that provide medical services (Moon et al., 2019; West et al., 2015).

*Corresponding author. E-mail address: razvan.corbos@man.ase.ro 
This paper has as main objective the identification of the main characteristics of the leaders from Colțea Clinical Hospital and the predominant leadership styles, in the conditions and context of the COVID-19 pandemic, but also the evaluation of interpersonal relations and medical services quality under these crisis conditions. The paper also covers a gap in the literature as it is a good time to notice any changes in leadership style, interpersonal relationships and the quality of the medical act in the context of an ongoing crisis such as the crisis created by the COVID-19 pandemic within medical units.

The paper is structured in four parts followed by conclusions and recommendations. After the introduction, the second part presents the results of works that had similar approaches and which represent the basis on which we built the research model. The third part of the paper addresses the research methodology including the methods and tools used, and in the fourth part we present the main results. The paper concludes with the main conclusions and a brief but comprehensive set of recommendations that can be useful to managers in organizations that provide medical services.

\section{LITERATURE REVIEW}

In general, hospitals have various areas of expertise being organized on several levels, both vertically and horizontally. Hospitals are institutions that provide very important medical services, which are often urgent, which means that the demand and supply of medical services cannot be fully controlled (Kiliç \& Keskin, 2019). Today, perhaps more than ever, given the context of the health crisis caused by COVID-19, the leadership qualities of the hospital manager become a very important factor for the management of such an institution.

Drucker (1996) defined the leader as a person who has followers, and Zaleznik (1992) added that the leader inspires creativity and can create conflict. As a concept, leadership can be a process through which two or more members of a group interact (Bass, 1990) using the art of influencing others to reach their full potential when involved in various tasks or projects (Northhouse, 2021).

Many authors (Sfantou et al., 2017) consider that effective leadership of health workers is particularly important to ensure the quality of medical services provided in an integrated manner. The question we ask ourselves in this way is whether the leadership style in some medical units influences the quality of the medical act. The literature has also tried to answer this question, with some studies identifying strong relationships between leadership styles and the quality of the medical act. Moreover, both from the perspective of patients and from the perspective of health care providers, leadership is considered to be an element of the hard core that underlies the provision of an integrated and well-coordinated health service. In this respect, there are quite a few publications that support the above statements. For example, Wong and Cummings (2013) identified positive relationships between effective leadership styles and a high level of patient satisfaction. Leadership style has been shown to be a factor with a strong influence on the quality of medical care, as shown in a study by Havig et al. (2011).

Page (2004) showed in a study how the transformational leadership style is closely correlated with the implementation of effective management, which implies the existence of a culture of patient safety and security. The implications of leadership in the condition of patients come more as indirect effects that refer to attracting and retaining medical professionals who have a much richer experience, but also by ensuring a stable work climate (Houser, 2000). In another study, Sfantou et al. (2017) tried to correlate the leadership style with certain quality indicators of the medical act, and the results indicated the existence of a strong relationship.

In a study by Casida and Pinto-Zipp (2008), transformational leadership was positively correlated with an effective organizational culture within medical units, while transactional leadership had a very low level of correlation. Also, the laissez-faire leadership style was negatively correlated with the efficiency of the organizational culture. Thus, the leadership styles that proved to be the most effective were those that described a collaborative management and a dynamic process, with 
multiple facets, such as the case of the transformational style. Another study (Al-Mailam, 2004) showed that the transformational leadership style was associated with a high level of employees' perception of leadership effectiveness.

Given the current context of the COVID-19 pandemic, this paper aimed to identify predominant leadership styles and assess interpersonal relationships and the quality of medical care under these crisis conditions.

\section{RESEARCH METHODOLOGY}

In order to effectively carry out this research, a first stage consisted in choosing the research method, respectively the research tool. Thus, we used a quantitative research method, the opinion poll, using the questionnaire as a tool. On the one hand, the questionnaire was constructed using the famous Multifactor Leadership Questionnaire (MLQ-6S), created by the authors Bass and Avolio (1996), and on the other hand variables were introduced that described the research sample and the quality of the medical act, through which we studied the perceptions of respondents as a whole. The questionnaire was built using Google Forms and distributed to senior medical staff (doctors and other senior medical staff) at Colțea Clinical Hospital, being completed online.

Once the research method was chosen and the research tool was built, in the second stage it was necessary to establish a representative sample at the level of the medical staff within the targeted medical unit. In order to establish a representative sample, the literature provides us with a variety of ways. In this research approach we chose to use the method of statistical tables developed by Krejcie and Morgan (1970). An essential condition necessary to use the method proposed by the two authors is that the statistical population to be between $10-1,000,000$ observation units. According to the management plan for 2018-2020, entitled Improving the planning and organization of health services at Colțea Clinical Hospital authored by Dr. Mircea Lupușoru (2017), senior medical staff has 114 employees, which is the statistical population for this research. Thus, according to the statistical tables proposed by the authors mentioned above, a representative sample for this statistical population is 86 respondents. Following the application of the questionnaire, 93 responses were received that were also considered valid, which means that the sample is representative for this research approach.

In a third stage, the answers collected through the applied research tool, were analyzed in total, but also in three significant groups and were interpreted through suggestive tables and graphs built using the Microsoft Office tools.

\subsection{Multifactor leadership questionnaire (MLQ-6S)}

The MLQ-6S questionnaire provided a description of the leadership style of the hierarchical superior for each group of respondents. This was made possible through 21 statements that described leadership styles. By choosing a number from 0 to 4 (where: 0 - Not at all; 1 - Quite rarely; 2 - Sometimes; 3 - Quite often; 4 - Frequently, almost always) the frequency with which each statement matched the hierarchical superior was identified. When words such as others were encountered among the statements, they had the meaning of subordinates, clients or members of the work team. It was also requested to the respondents to assess the direct hierarchical superior and to consider exclusively the period of the COVID-19 pandemic.

The MLQ-6S measures leadership style based on seven factors related to transformational leadership. The score obtained for each of the seven factors is determined by summing the scores of three specific items in the questionnaire. For example, in order to determine the score for the first factor called idealized influence, the scores obtained in items 1,8 and 15 will be summed, following this procedure accordingly for each factor.

In short, the interpretation of the scores is done as follows: 
A score is considered high if you get a score between 9-12, moderate, if you get a score between 58 and low if you get a score between 0-4. The seven factors on which this analysis is performed are: - Factor 1 - idealized influence indicates whether the leader manages to gain and maintain the trust and respect of subordinates, shows them that he is dedicated to them, appeals to their hopes and dreams, acting as a true mentor;

- Factor 2 - inspirational motivation measures the degree to which the leader provides a vision, uses appropriate symbols and images to help others focus on their work, trying to make others feel that their work is meaningful;

- Factor 3 - intellectual stimulation shows the degree to which the leader encourages others to be creative in looking at old problems in new ways, to create a tolerant environment with seemingly extreme positions, and urges people to doubt their own values and beliefs in relation to those of the organization;

- Factor 4 - individualized consideration indicates the degree to which the leader shows interest in the well-being of others, assigns individual projects and pays attention to those who seem less involved in the group;

- Factor 5 - contingent reward shows the degree to which the leader tells others what to do to be rewarded, emphasizes what he expects from them and recognizes their achievements;

- Factor 6 - management-by-exception evaluates the extent to which the leader communicates the requirements of the job to others, is satisfied with a standard performance and strongly believes in the following motto: "if it is not broken, do not fix it.";

- Factor 7 - laissez-faire measures whether the leader asks little of subordinates, is content to let things go by themselves, and lets others do what they see fit.

The MLQ-6S questionnaire evaluates both the transactional leadership style, through factors 5, 6 and 7 , and the transformational leadership style, through factors 1, 2, 3 and 4.

Transactional leaders start from the following premises: employees are motivated through rewards and punishments; subordinates must obey the orders of the superior; subordinates do not selfmotivate and must be closely monitored and controlled to perform their tasks.

On the other hand, transformational leaders differ from others because: they encourage motivation and positive development; exemplifies moral standards within the organization and encourages others to do the same; promotes an ethical work environment, with clear values, priorities and standards; emphasizes authenticity, cooperation and open communication; provides coaching and guidance, but allows employees to make decisions and take ownership of tasks.

\section{RESULTS}

\subsection{MLQ-6S results at Colțea Clinical Hospital during the COVID-19 pandemic}

Table 1 shows the results of applying the MLQ-6S questionnaire. Thus, we can notice the fact that in the Colțea Clinical Hospital, a certain leadership style does not predominate, whether we are talking about the transactional one or the transformational one. Both at the level of the whole sample and at the level of groups, the results are balanced, the scores obtained being mostly moderate. Thus, at the level of the medical unit we are dealing with a combination of the two styles, a fact confirmed by many other studies from different industries. However, it should be noted that most of the answers, whether we look at them as a whole or in groups, tip the scales in favor of a rather transformational leadership style. 
Table 1. MLQ-6S results by total and by groups

\begin{tabular}{|c|c|c|c|c|c|c|c|c|c|c|c|c|}
\hline \multirow{2}{*}{ Factors } & \multirow{2}{*}{$\begin{array}{c}\text { Results/ } \\
\text { total }\end{array}$} & \multicolumn{2}{|c|}{ Results/position level } & \multicolumn{4}{|c|}{ Results/occupied position } & \multicolumn{5}{|c|}{ Results/academic degree } \\
\hline & & $\begin{array}{c}\text { Managing } \\
\text { position }\end{array}$ & $\begin{array}{c}\text { Execution } \\
\text { position }\end{array}$ & $\begin{array}{c}\text { Other } \\
\text { senior } \\
\text { health } \\
\text { staff }\end{array}$ & $\begin{array}{l}\text { Primary } \\
\text { care } \\
\text { physician }\end{array}$ & $\begin{array}{l}\text { Resident } \\
\text { physician }\end{array}$ & Specialist & $\begin{array}{l}\text { Assistant } \\
\text { Professor }\end{array}$ & Lecturer & $\begin{array}{l}\text { Associate } \\
\text { Professor }\end{array}$ & Professor & $\begin{array}{l}\text { Not } \\
\text { the } \\
\text { case }\end{array}$ \\
\hline $\begin{array}{l}\text { Idealized } \\
\text { influence }\end{array}$ & 8 & 7 & 8 & 8 & 7 & 8 & 7 & 6 & 7 & 7 & 10 & 8 \\
\hline $\begin{array}{c}\text { Inspirational } \\
\text { motivation }\end{array}$ & 8 & 9 & 8 & 11 & 7 & 9 & 7 & 6 & 7 & 6 & 10 & 9 \\
\hline $\begin{array}{l}\text { Intellectual } \\
\text { stimulation }\end{array}$ & 7 & 8 & 7 & 11 & 7 & 9 & 6 & 4 & 6 & 5 & 11 & 8 \\
\hline $\begin{array}{c}\text { Individualized } \\
\text { consideration }\end{array}$ & 7 & 8 & 7 & 11 & 7 & 9 & 7 & 6 & 7 & 5 & 10 & 8 \\
\hline $\begin{array}{c}\text { Total } \\
\text { transformational } \\
\text { (average) }\end{array}$ & 7.5 & 8 & 7.5 & 10.25 & 7 & 8.75 & 6.75 & 5.5 & 6.75 & 5.75 & 10.25 & 8.25 \\
\hline $\begin{array}{c}\text { Contingent } \\
\text { reward }\end{array}$ & 6 & 7 & 6 & 10 & 6 & 8 & 5 & 4 & 5 & 3 & 7 & 7 \\
\hline $\begin{array}{l}\text { Management-by- } \\
\text { exception }\end{array}$ & 9 & 10 & 9 & 12 & 9 & 10 & 9 & 8 & 8 & 7 & 12 & 10 \\
\hline Laissez-faire & 7 & 7 & 6 & 6 & 7 & 7 & 6 & 7 & 6 & 6 & 9 & 7 \\
\hline $\begin{array}{c}\text { Total } \\
\text { transactional } \\
\text { (average) }\end{array}$ & 7.33 & 8 & 7 & 9.33 & 7.33 & 8.33 & 6.66 & 6.33 & 6.33 & 5.33 & 9.33 & 8 \\
\hline
\end{tabular}

*red = high score; yellow = moderate score

Source: authors based on research results 
In order to customize the results and better understand how the two leadership styles intertwine within this organization, we can look at the factors with the highest scores, both for transformational and for transactional. Thus, we notice that on the transformational leadership side, the factors with the highest scores were the idealized influence and the inspirational motivation, both obtaining moderate scores. On the transactional leadership side, a high score was obtained by the management-by-exception factor. Next, we will make a description of these factors and how they influence the relationship between leader and subordinates, thus outlining the main characteristics of the leaders in the Coltea Clinical Hospital.

Idealized influence involves leaders who believe in the philosophy that a leader can only influence his subordinates when he/she applies what he/she communicates to others. These leaders act as mentors that subordinates seek to imitate. Such leaders gain the trust and respect of subordinates through their actions. They usually place the needs of subordinates before their own needs, sacrifice their personal earnings for the earnings of subordinates, and have high ethical standards. They use the power they have to influence their subordinates to orient themselves towards the common goals of the organization.

Inspirational motivation is at the core of transformational leadership and consists in promoting a consistent vision, mission and set of values. Their vision is so convincing that they know very clearly what they want as a result of each interaction. Transformational leaders lead their subordinates by giving them a sense of significance, but also of challenge. They enthusiastically and optimistically build a team spirit and a sense of commitment.

Management-by-exception is a characteristic of the transactional leader, which intervenes when the standards imposed by him/her are not met or when the performance of subordinates is below his/her expectations. They use punishment as a corrective tool in response to the poor performance of subordinates.

Therefore, the characteristics presented above are the defining ones for the leaders within the Coltea Clinical Hospital, according to the results of the application of the MLQ-6S questionnaire. Thus, we notice the almost balanced presence of transformational and transactional leadership, which outlines a number of advantages and disadvantages that we will mention below.

As disadvantages, transformational leadership, as a theory to be applied, is very difficult to be trained or taught because it is a combination of many other leadership theories. Also, some subordinates could be manipulated by such leaders and there is a chance that they will lose more than gain. However, the advantages of transformational leadership are very well seen in the current environment, characterized by uncertainty, global turbulence and organizational instability. The subordinates of such leaders show quite high levels of job satisfaction and organizational commitment, creating a devoted workforce.

The disadvantage of transactional leadership can sometimes be that it pays too much attention to very detailed, short-term goals, following a set of standard rules and procedures. As an advantage, however, this type of leadership style can work well when the organization's problems are simple and clearly defined. Transactional style is often considered insufficient, although it is seen as good enough to develop the maximum potential of leadership. This is the foundation for more mature future interactions, but leaders will need to be careful not to practice this style alone in order to avoid creating an organizational environment driven by power, status, and individual advantage.

\subsection{Interpersonal relations during the COVID-19 pandemic at Colțea Clinical Hospital}

After determining the leadership styles and its main characteristics within the Colțea Clinical Hospital during the COVID-19 pandemic, the research process continued by evaluating the current leadership style compared to the pre-pandemic period and current and future interpersonal relationships. In this regard, three questions were developed, the results of which are presented through suggestive graphs. 


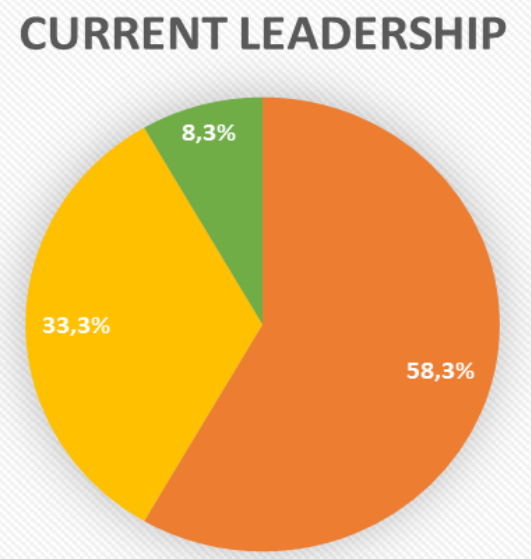

Figure 1. Perceptions of current leadership

Source: authors, based on research results

At the level of the entire research sample, the question "How do you assess, as a whole, the current leadership compared to the one before the COVID-19 pandemic?" returned the following results: better 33.3\%; unchanged 58.3\%; worse $8.3 \%$ (see Figure 1).

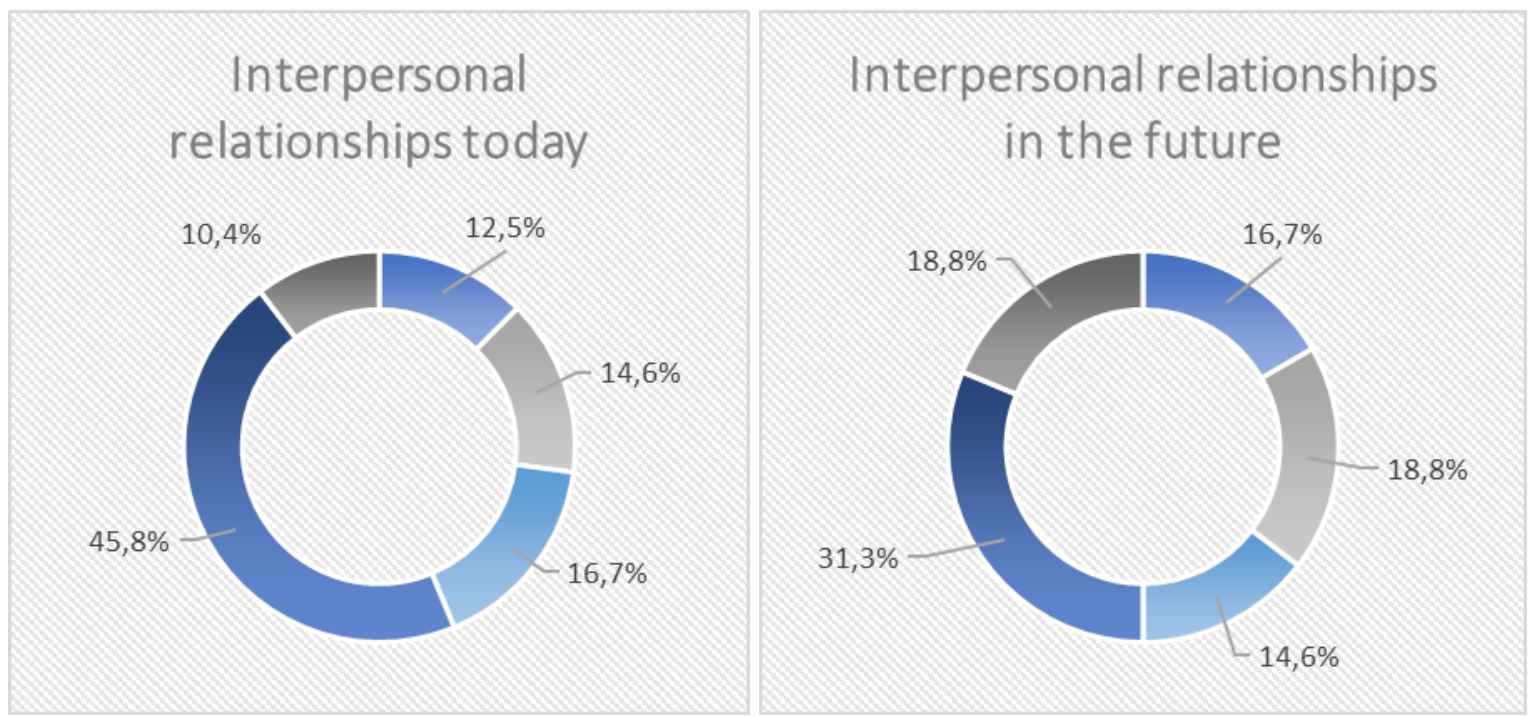

Figure 2. Perceptions on present and future interpersonal relationships

Source: authors, based on research results

Perceptions of interpersonal relationships have been studied for both the present and the future. In this sense a scale from 1 to 5 was used, where $1=$ Not at all; $5=$ To a very large extent.

Thus, at the level of the entire sample, the statements "I consider that interpersonal relations are currently closer than before the COVID-19 pandemic." "I believe that in the future, human relations will improve." revealed that both now and in the future, interpersonal relations have improved, and will improve, to a weighted extent. (See Figure 2).

\subsection{The quality of the medical act during the COVID-19 pandemic at the Colțea Clinical Hospital}

The last part of the research approach consisted in the study of perceptions regarding the quality of the medical act during the COVID-19 pandemic. In this sense, in the questionnaire were introduced 8 items that evaluate the quality of the medical act, as follows: 
- The hospital develops and implements a policy to ensure and improve patient safety.

- Communication with the patient aims at its involvement in the care process.

- Safety: The aim is to avoid accidents in the patients care process, which was created to help them.

- Effectiveness: Provides medical services based on scientific evidence and best medical practice.

- Patient placed in the center of care: The provision of care respects and responds to the preferences, needs and values of each patient, ensuring that patient values guide all clinical decisions.

- Providing timely care: The aim is to reduce waiting times and delays, which are sometimes detrimental to both those who receive and those who provide medical care.

- Efficiency: The aim is to avoid losses, including those caused by equipment damage, improper use of supplies, ideas and energy.

- Equity: The provision of care services does not vary in quality due to personal characteristics, such as gender, nationality, geographical location and socio-economic status.

To assess the perception of the quality of the medical act during the Covid-19 pandemic, for the 8 items stated above, a scale from 1 to 5 was used, where $1=$ total disagreement and $5=$ total agreement. Thus, both at the level of the entire research sample and at the level of groups, the results follow the same trend in terms of the quality of the medical act during the COVID-19 pandemic. All results are positive, with small variations in the variables that describe the efficiency of medical services and the care provided on time, depending on the group of respondents, but without significant differences. The highest scores were obtained by the equity of medical services and the item that described ensuring and improving patient safety, elements that can characterize the activity of medical units during the COVID-19 pandemic.

\section{CONCLUSION AND RECOMMENDATIONS}

The research approach on the leadership style, interpersonal relations and the quality of the medical act during the COVID-19 pandemic, within the Coltea clinical hospital resulted in the identification of the main characteristics of the leaders within the medical unit, the manifestation of interpersonal relations, but also the degree to which the quality of the medical act was maintained in this period of crisis.

From the point of view of the leadership style, the research revealed the fact that within the medical unit a rather transformational leadership style was manifested, with very small transactional influences. Thus, the main characteristics of the leaders of the Colțea Clinical Hospital are reduced to mentor behaviors that promote a certain vision, accompanied by a mission and a set of values, giving a sense of significance and challenge to subordinates. Also, another characteristic of these leaders is that they apply punishment as a corrective tool, when subordinates perform poorly. Regarding the current leadership style, it is largely seen as unchanged from the pre-pandemic period, and perceptions of interpersonal relationships are positive, in the sense that most respondents believe that both now and in the future these relations have improved, respectively, are about to improve. All these aspects denote a strong team spirit, which can be attributed to the applied leadership styles.

From the point of view of the quality of the medical act, the highest scores were obtained by the equity of medical services and the item that described ensuring and improving patient safety, elements that can characterize the activity of medical units during the COVID-19 pandemic because they were paid more attention. Overall, the quality of the medical act was maintained. 
Based on these conclusions, we made the following recommendations:

- The leadership style within the medical unit has both transformational and transactional tendencies, so a first recommendation would be for leaders to be aware of this aspect in order to evaluate their own behaviors, and in this sense, to organize an evaluation at least annually;

- Given that the transformational leadership style is closely linked in a positive way to the quality of the medical act, but also to the employees' perception of interpersonal relationships, hospitals could benefit from the recruitment of transformational leaders;

- Encouraging team spirit by organizing events dedicated to employees outside of working hours, in order to strengthen relations between members of work teams;

- Organizing training courses and modules for employees on exploring transformational and transactional leadership styles could lead to a deeper understanding of the elements of effective leadership in the hospital;

- Increasing the quality of the medical act mainly by providing timely care and by involving the patient in the care process through better communication.

The current research is not without limitation. One major setback is that the results are only representative for one institution and they cannot be generalized. One way to reduce this limitation is to apply the research method in other similar organizations and to compare the results. As future research directions one may apply the questionnaire also in privately owned and operated medical organizations in order to identify differences between leadership styles based on being a public or private organization.

\section{REFERENCES}

Al-Mailam, F. F. (2004). Transactional versus transformational style of leadership-employee perception of leadership efficacy in public and private hospitals in Kuwait. Quality Management in Healthcare, 13(4), 278-284.

Al-Sawai, A. (2013). Leadership of healthcare professionals: where do we stand? Oman medical journal, 28(4), 285.

Bass, B. M. (1990). From transactional to transformational leadership: Learning to share the vision. Organizational dynamics, 18(3), 19-31.

Bass, B. M., \& Avolio, B. J. (1996). Multifactor leadership questionnaire. European Journal of Psychological Assessment. https://doi.org/10.1037/t03624-000

Casida, J. J., \& Pinto-Zipp, G. (2008). Leadership-organizational culture relationship in nursing units of acute care hospitals. Nursing Economics, 26(1), 7.

Drucker, P. F. (1996). Your leadership is unique. Leadership, 17(4), 54.

Havig, A. K., Skogstad, A., Kjekshus, L. E., \& Romøren, T. I. (2011). Leadership, staffing and quality of care in nursing homes. BMC Health Services Research, 11(1), 1-13.

Houser, J. L. (2000). A model for evaluating the context of nursing care delivery. University of Northern Colorado.

Kiliç, T., \& Keskin, S. (2019). What Type of Leadership Is Suitable for Hospital Management? In Proceedings of the INTERNATIONAL MANAGEMENT CONFERENCE (Vol. 13, No. 1, pp. 806-810). Faculty of Management, Academy of Economic Studies, Bucharest, Romania.

Krejcie, R. V., \& Morgan, D. W. (1970). Determining sample size for research activities. Educational and psychological measurement, 30(3), 607-610.

Lupușoru, M. (2017). Îmbunătățirea planificării și organizării serviciilor de sănătate la nivelul Spitalului Clinic Colțea. Retrieved 28 August 2021, from: https://www.coltea.ro/wpcontent/uploads/2017/06/Proiect-management-Spitalul-Clinic-Coltea2018.pdf. 
Moon, S. E., Van Dam, P. J., \& Kitsos, A. (2019, December). Measuring transformational leadership in establishing nursing care excellence. In Healthcare (Vol. 7, No. 4, p. 132). Multidisciplinary Digital Publishing Institute.

Northouse, P. G. (2021). Leadership: Theory and practice. Sage publications.

Page, A. E. (2004). Transforming nurses' work environments to improve patient safety: The Institute of Medicine recommendations. Policy, Politics, \& Nursing Practice, 5(4), 250-258.

Sfantou, D. F., Laliotis, A., Patelarou, A. E., Sifaki-Pistolla, D., Matalliotakis, M., \& Patelarou, E. (2017, December). Importance of leadership style towards quality-of-care measures in healthcare settings: a systematic review. In Healthcare (Vol. 5, No. 4, p. 73). Multidisciplinary Digital Publishing Institute.

West, M., Armit, K., Loewenthal, L., Eckert, R., West, T., \& Lee, A. (2015). Leadership and leadership development in health care: the evidence base. Retrieved 29 August 2021, from: https://www.kingsfund.org.uk/sites/default/files/field/field_publication_file/leadershipleadership-development-health-care-feb-2015.pdf.

Wong, C. A., Cummings, G. G., \& Ducharme, L. (2013). The relationship between nursing leadership and patient outcomes: a systematic review update. Journal of nursing management, 21(5), 709-724.

Zaleznik, A. (1992). Managers and leaders: are they different? Harvard Business Review, 70(2), 126-135. 